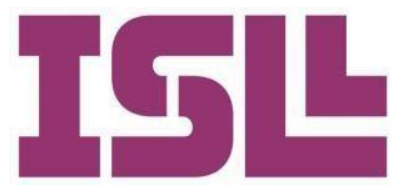

Número 10

Diciembre de 2018

\title{
La inteligencia emocional como predictor del hábito lector y la competencia lectora en universitarios
}

\author{
Elena Jiménez Pérez \\ Universidad de Jaén
}

Recibido: 01-07-2018

Aceptado: 30-09-2018

Pág. 30 a la 54

\section{Palabras clave \\ Competencia lectora, inteligencia emocional, universitarios, hábitos lectores, leer}

\section{Resumen:}

En el presente estudio se analiza la relación entre inteligencia emocional y competencia lectora a partir del hábito lector en un total de 1051 estudiantes universitarios andaluces para comprobar si los alumnos que puntúan más alto en hábitos lectores son los que presentan mejores resultados en competencia lectora y si está relacionado con una mejor inteligencia emocional. Para llevar a cabo esta investigación se han utilizado herramientas testeadas: el cuestionario de hábitos lectores del MECD, el test de competencia lectora ComLec y el test de Inteligencia Emocional Wong and Lang (2002). Las TIC han sido la forma de presentación de los test al alumnado mediante un enlace web con criterios de exclusión para aquellos cuestionarios que pudieran resultar nulos. Los resultados apuntan a que los discentes que puntúan mejor en inteligencia emocional, más felices según Wang y Lang, muestran una competencia lectora mayor y entienden la lectura como parte de sus vidas, lo que puede establecer una relación natural entre los tres parámetros, y abre nuevos horizontes de investigación para determinar más detalladamente en qué consiste esa relación y cómo mejorar una entrenando otras. Como conclusión se puede extraer que la relación entre el hábito lector, la competencia lectora y la inteligencia emocional es directamente proporcional. 
Esta investigación se llevó a cabo en el marco de la estancia de investigación en la Universidad de Alicante realizada por la autora, en el departamento de Didáctica de la lengua y la literatura, bajo la supervisión del Dr. Díez Mediavilla.

\section{Introducción}

Si se atiende a los informes del Gremio de Editores de España, donde se señala un descenso en la compra de libros en general que no rompe la tendencia negativa de ventas desde el 2008, cabe destacar que en el país cada vez se leen menos libros. Así, se entiende que cada vez sean más los profesores universitarios que se lamentan de que cada vez se lee menos (García, 2011), cuestión que es admitida por los propios alumnos (Gilardoni, 2006) y, por extensión, que la comprensión lectora no ha mejorado en los últimos 3 lustros (Ripoll y Aguado, 2014) ya que existe una estrecha relación entre hábito lector y competencia lectora según la OCDE. Los alumnos universitarios acceden a la carrera ya con deficiencias para comprender los textos (Calderón y Quijano, 2010) y otras debilidades en comprensión lectora (Domínguez y Pérez, 2009), que van unidas a su escaso interés por la lectura a favor de otras formas de entretenimiento (Gilardoni, 2006). Dicha información contrasta con las encuestas oficiales de hábitos lectores que se han venido realizando por el Observatorio Andaluz de la Lectura (VV. AA., 2011), por ejemplo, donde el 70.5\% de los andaluces encuestados con edades comprendidas entre 14 y 24 años afirman leer de forma habitual. Posiblemente, más de la mitad de los sujetos distorsiona su imagen con las medidas clásicas de lectura por "la deseabilidad social" de pertenecer a la clase lectora (Larrañaga y Yubero, 2005), ya que esta se puede entender como elitista porque ser lector requiere cierto nivel de autoexigencia y disciplina lectora.

Pero el estudiante universitario, más allá de sus consideraciones, debe ser un buen lector de verdad (Cisneros, Olave y Rojas, 2012) y no usar la lectura exclusivamente para leer apuntes (Teberosky et al., 1996). Y, sobre todo, los universitarios deben tomar consciencia de si comprenden lo que leen realmente (González et al., 2010 para que ellos mismos disciernan si deberían mejorar su competencia lectora.

Cabe destacar que los adolescentes con mayor motivación hacia la lectura, entendida como hábito lector consolidado, tienen mejor competencia lectora e identifican más rápido las webs con información útil (Moje et al., 2008. También que las personas con mejor gestión emocional necesitan menos energía para tramitar información (Dávalos y León, 2013), por lo que existe la posibilidad de dedicar más tiempo a otras formas de ocio y, por tanto, ser más felices.

El peso de la inteligencia emocional es muy importante en la vida académica (Gutiérrez y Fernández-Castillo, 2009), ha quedado demostrado en estudios (Petit, 2003; Castellano, 2010; De Nóbrega y Franco, 2014). Algunos autores se inclinan a que la inteligencia emocional posibilita alcanzar un rendimiento adecuado en competencia lectora por cómo se relaciona con la regulación de la percepción a través de la comprensión de los sentimientos, la capacidad de aprendizaje según el estado de ánimo 
(Villardón-Gallego et al., 2013). Lo que realmente es significativo de la inteligencia emocional para este estudio es que su correcta gestión confiere mayor responsabilidad al individuo, mejor capacidad de concentración, y de prestar atención a la tarea que se está llevando a cabo, menor impulsividad y mejor autocontrol (Goleman, 2007), todos ellos componentes facilitadores de la tarea lectora. Esto, en parte, podría explicar la relación de la baja tasa de fracaso escolar en alumnos con hábito lector más consolidado según la OCDE, si existe relación entre los constructos de inteligencia emocional y competencia lectora con referencia a los hábitos de lectura.

En cuanto a la comprensión lectora, entendida como la capacidad de un ser humano de entender lo que ha querido decir un autor mediante un texto escrito lo más objetivamente posible (Jiménez-Pérez, 2014), se establece que el proceso de comprender un texto cuando se lee enciende o estimula las emociones - ya sean positivas o negativas - casi al mismo tiempo (Dávalos y León, 2013), por lo que ya se apunta teóricamente a que existe una relación entre comprender un texto y la inteligencia emocional de un sujeto. Sin olvidarse de que el acto de leer, como forma natural de entrenar la competencia lectora (Jiménez-Pérez, 2014), produce sensaciones provechosas en el organismo (Dávalos y León, 2013) en forma de percepción positiva de las emociones (Moje et al., 2008). Puesto que existe una correlación entre la inteligencia emocional y la verbal (Ferrando, 2006), se explicaría una relación directa también con la competencia lectora, ambas estrechamente relacionadas según Gardner (1993) en su teoría de las inteligencias múltiples, esto es, la habilidad de un ser humano de utilizar su comprensión lectora de forma útil en la sociedad que le rodea (Jiménez- Pérez, 2014).

El objetivo principal de esta investigación es realizar un análisis descriptivo de la distribución de la inteligencia emocional, por una parte, y de la competencia lectora, por otra, según los hábitos lectores. Así pues, como hipótesis, se parte de la posibilidad de que los alumnos con hábitos lectores más consolidados deberán tener mejor competencia lectora. Por eso, se plantea la posibilidad de que las puntuaciones de los tres parámetros estén vinculadas de forma directamente proporcional. En este sentido abogamos por la tesis de que existe una relación entre hábitos lectores con respecto a la inteligencia emocional y la competencia lectora.

\section{Método}

\section{Participantes}

En total, los 1051 alumnos que han realizado el test se dividen según el sexo en 474 hombres y 577 mujeres. Lo que arroja unos porcentajes de $45.09 \%$ de hombres y $54.91 \%$ de mujeres. El hecho de que el número de mujeres sea mayor puede ser debido a la prevalencia de departamentos de Didáctica donde se imparte docencia en carreras 
de Grado de Infantil, Grado de Primaria y Máster de Secundaria, donde el número de mujeres es superior al de hombres.

El apartado de los test del presente estudio ha sido diseñado informáticamente para facilitar el acceso a los cuestionarios al máximo número de alumnos posible y favorecer la labor a los profesores que se han prestado a participar en este estudio, evitando así el uso de cuestionarios tradicionales en papel para agilizar los trámites. Además, los encuestados saben si han terminado el cuestionario correctamente y sin incidencias porque al llegar al final se publica un código personal que confirma la conclusión del test global y da fe de que la información facilitada se ha pasado satisfactoriamente a la base de datos.

Para validar los cuestionarios realizados, existen varios matices programados que invalidan automáticamente el test provocando que los datos que se graben no se pasen a la base de datos principal. Para empezar, el tiempo lógico de cada bloque está estipulado de antemano por el estudio base que respalda cada test; por ejemplo, el de inteligencia emocional se estima en 8 minutos, por lo que todo aquel test que exceda en el triple ese tiempo es anulado (es decir, un test de IE de 24 minutos en adelante es borrado automáticamente, y con él, el resto de la información vinculante). En este caso, el límite inferior se señala en una cuarta parte, es decir, todo aquel test que requiera menos de 2 minutos no se tendrá en cuenta. El test de hábitos lectores requiere un tiempo de 15 minutos de media y el de competencia lectora 30 minutos, y a ambos se le aplica el mismo sistema de descalificación que al anterior. Para terminar, cada test - dividido en los tres bloques anteriormente señaladas (IE, CL y HL) - que arroje un porcentaje de cinco respuestas repetidas seguidas superior al 50\% no es guardado en la base de datos; de igual forma ocurre con las respuestas en blanco. Cada test computa el mismo porcentaje e impide que se guarde en la base de datos final.

\section{Instrumentos}

El cuestionario definitivo ha sido planteado con base en tres test:

Tabla 1. Instrumentos de evaluación de los ítems

\begin{tabular}{ccc}
\hline HL $\left(15^{\prime}\right)$ & IE (8') & CL (30’) \\
\hline Encuesta de hábitos & Encuesta de & Encuesta de \\
lectores de 2001 & Inteligencia Emocional & Competencia Lectora \\
publicada por el & de Wong y Lang & ComLec \\
MECD & & \\
& & \\
\hline
\end{tabular}




\section{El cuestionario del MECD sobre Hábitos Lectores de la población escolar}

El cuestionario del Ministerio de Educación Ciencia y Deporte de España, generado en 2001, está compuesto de 50 preguntas, enfocado para un público general disperso por todo el territorio español (por lo que presta atención a otras lenguas maternas) que abarca a más de 12.000 usuarios encuestados, alumnos escolarizados a partir de 15 años en adelante.

Las primeras 11 preguntas, mediante subapartados que amplían aritméticamente el espectro que analizar, recopilan información personal del alumnado, desde la edad o su lengua materna hasta su uso de internet, número de baños de su hogar o estudios de los padres para establecer un perfil socio-económico del encuestado.

A partir de la pregunta número 12, el interés se centra en el círculo académico del alumnado (notas, asignaturas, expectativas, centro escolar...).

De la pregunta 15 a la 50, incluyendo sus subítems, el cuestionario se centra en analizar el hábito lector per se y en relación con otras actividades. Es la parte más importante de la encuesta en lo referente a obtener información objetiva sobre el hábito de leer y también sondea el papel que juega el profesor en inculcar dicha rutina.

La entidad encargada de realizar la encuesta, el Ministerio de Educación, Ciencia y Deporte, que suele valerse del INE (Instituto Nacional de Estadística), es uno de los órganos más solventes en proyección, recogida y análisis de datos que, en ocasiones, sirve de punto de partida para estudios avalados por el CSIC.

Con respecto a la categorización de los hábitos lectores, de ese mismo cuestionario se han seleccionado ocho ítems (preguntas 13, 19, 21, 24, 28, 36, 50) que responden a las siguientes cuestiones: cuánto leen los padres o tutores, si la lectura es un tema de discusión cotidiano en el ambiente familiar, si el individuo suele leer en su tiempo libre o lo dedica a otras actividades prioritarias, cuántos libros hay en casa sin contar los de texto (considerados obligatorios), si el motivo principal por el que el encuestado lee es el placer u otro, y si está de acuerdo con una serie de afirmaciones correspondientes a la lectura. Con esta selección se ha creado un algoritmo para obtener tres tipos de hábitos lectores: bajo, medio y alto, siendo la lectura por placer el punto de partida (se obvian las lecturas escolares, académicas, etc.). Se han valorado estos ítems positivamente cuando muestran, al menos, un $50 \%$ de respuestas que tienden al interés en la lectura en aquellas cuestiones que contemplan varios subapartados, por ejemplo, se valora positivamente elegir "porque me gusta" en vez de "porque me obligan" en respuesta a por qué leen.

De esta manera, los hábitos lectores bajos corresponden a aquellos alumnos que responden de forma positiva a 3 o menos ítems de los 8 anteriormente señalados, los 
hábitos lectores medios cuando responden de forma positiva a 4 ítems y alto cuando responden de forma positiva a más de 5 ítems, de los seleccionados.

\section{El test de inteligencia emocional WONG y LANG (2002)}

El nombre completo de este test es Wong and Law Emotional lntelligence Scale (WLEIS) y es una medida de autoinforme básica de Inteligencia Emocional. El autor es el Dr. Chi-Sum Wong (Department of Management The Chinese University of Hong Kong Shatin, en Hong Kong, China). Carece de adaptaciones al castellano y su descripción del tipo de evaluación se centra en ser una medida de autoinforme compuesta por 16 ítems con una escala tipo Likert de 7 puntos donde, en general, a mayor puntuación mayor inteligencia emocional. En cuanto a los objetivos de esta evaluación, se trata de un registro de la inteligencia emocional en el ámbito organizacional. Los factores evaluados en este test se miden en una escala que está compuesta por cuatro dimensiones (cuatro ítems por dimensión): 1) Evaluación de las propias emociones; 2) Evaluación de las emociones de los demás; 3) Uso de las emociones, y 4) Regulación de las emociones. La edad para realizar este test es a partir de los 16 años en adelante mientras que el tiempo de administración fluctúa entre los cinco y los diez minutos. Los datos psicométricos están operados a través del alfa de Cronbach y la consistencia interna informada fue que la evaluación de las propias emociones ascendía a $\alpha=0,87$, entre los que destaca la percepción de la propia felicidad; la evaluación de las emociones de los demás se posicionaba en $\alpha=0,90$ donde se establece la capacidad de empatizar con los demás. Así el uso de las emociones en $\alpha=0,84$ como capacidad de positivizar el día a día o gestionar el estrés; y la regulación de las emociones en $\alpha=0,83$ como forma de autocontrol y autoconocimiento.

\section{El test de competencia lectora ComLec}

El test ComLec (Llorens et al., 2011) está compuesto por cinco textos, tres continuos y dos discontinuos y un total de 20 preguntas elaboradas siguiendo los parámetros PISA 2000. La extensión de los textos varía de las 274 palabras a las 426 y son escritos fundamentalmente expositivos y argumentativos, en lo referente a los continuos, y diagrama y gráfico con o más de 130 palabras los discontinuos.

Las veinte preguntas se dividen en recuperación de información, integración, reflexión sobre el contenido y la forma del texto. El formato de respuesta se divide en elección múltiple con cuatro alternativas. Las preguntas son de tipo abiertas y cerradas predominando las de elección múltiple porque esta prueba ha sido diseñada con intención de programarla y así facilitar su corrección. Por ese motivo, las preguntas abiertas dificultarían esta finalidad al no poder cercar del todo cierto tipo de preguntas desde un punto de vista de la lingüística computacional. 
Los datos psicométricos también están evaluados según el coeficiente alfa de Cronbach donde se obtuvo un índice global de ,795. Se muestran los índices de dificultad de los ítems (valores entre 25 y ,74) y también aparecen los índices de homogeneidad de los ítems (valores entre $, 21 \mathrm{y}, 48$ ).

A pesar de que este test ha sido ideado para adolescentes de 15 años los textos y las preguntas que se han utilizado son perfectamente válidos para rangos de edad superiores ya que se han usado el tipo de textos y de preguntas sugeridas por PISA tanto para alumnos escolarizados como para adultos.

El planteamiento del test deja a criterio del sujeto si leer el texto entero para empezar o, por el contrario, las preguntas, por ejemplo. Por eso, se pide a los estudiantes que utilicen la información del texto para responder a las preguntas dejándoles la posibilidad de utilizar sus propias estrategias.

El test de competencia lectora usado plantea una tabla de centiles arrojados por el estudio de validez del proyecto, que se va a tomar solo como referencia para el presente análisis. Por lo tanto, aunque sirva como punto de partida la media obtenida por los alumnos de 15 años, esta información va a venir definida por la media que obtengan los propios estudiantes universitarios al realizar el test de competencia lectora.

\section{Procedimiento}

Se han escogido a los alumnos universitarios partiendo de una edad base lógica, los 18 años, edad oficial con la que un estudiante no repetidor puede acceder a la enseñanza superior y dando una amplitud de arco de diez años por la tendencia en la actualidad a no terminar los estudios en el tiempo de graduación mínima establecida por ley, y por la tendencia a complementar los estudios con idiomas, másteres, etc.

Se ha tenido en cuenta a ambos sexos en la medición de hábitos lectores, inteligencia emocional y competencia lectora pero se han descartado los test de estudiantes Erasmus por la probabilidad de no dominar el idioma lo suficiente.

Este estudio se basa en los datos de investigación recogidos en un cuestionario que ha estado publicado en internet en 2014. La dirección ha estado abierta en la página web http://testcl.comprensionlectora.es durante más de 8 meses con la intención de llegar al profesorado universitario que ha colaborado para que sus alumnos realizaran dicho cuestionario. El test se ha compuesto de varios bloques, de los que se han extraído los correspondientes a IE (inteligencia emocional), HL (hábitos lectores) y CL (competencia lectora), además del bloque de datos personales, que agrupan un total de 203 preguntas. De estos 203 ítems: 
-159 pertenecen al primer cuestionario, que se corresponde al de hábitos lectores y que integran las preguntas con sus subapartados.

-16 al segundo cuestionario, cuya correspondencia es la inteligencia emocional y se mantiene sin adaptaciones por lo que para la obtención de la puntuación se respeta las pautas originales.

-24 al de competencia lectora, que también permanece fiel al cuestionario original. -Ítems referentes a edad, sexo, centro de estudios y nivel.

\section{Resultados}

En primer lugar, se analizan los valores medios de edad con respecto a los hábitos lectores. La edad media de los universitarios ronda los 21 años, siendo los alumnos con mejor hábito lector los más jóvenes, con una media de edad de 20.97.

Tabla 2. Hábito Lector y Media de Edad

\begin{tabular}{lccccr}
\hline HL & Edad & Desv.típ. & Mínimo & Máximo & $\%$ \\
Bajo & 21,65 & 1,729 & 18 & 24 & $31,7 \%$ \\
Medio & 21,08 & 1,625 & 18 & 24 & $57,0 \%$ \\
Alto & 20,97 & 1,579 & 18 & 24 & $11,3 \%$ \\
TOTAL & 21,24 & 1,673 & 18 & 24 & $100,0 \%$ \\
\hline
\end{tabular}

Si se observa la tabla, se puede extraer que una mayoría de universitarios encuestados entran en la categoría de hábito lector medio, con un $57 \%$ del total en puntuación 1. Es significativo destacar que solo un 11,5\% presentan hábitos lectores sólidos, puesto que cabría esperar que los universitarios pudieran presentar hábitos lectores más consolidados.

Tabla 3. Hábito Lector y Media de Competencia Lectora

\begin{tabular}{|c|c|c|c|c|c|}
\hline HL & Media CL & Desv.típ. & Mínimo & Máximo & $\%$ \\
\hline Bajo & 12,887 & 2,2900 & 7,0 & 19,0 & $19,5 \%$ \\
\hline Medio & 14,130 & 2,7045 & 6,0 & 19,0 & $65,2 \%$ \\
\hline Alto & 17,651 & 1,0410 & 13,0 & 19,0 & $15,3 \%$ \\
\hline $\begin{array}{l}\text { TOTAL } \\
100,0 \%\end{array}$ & 14,425 & 2,8384 & & & 19,0 \\
\hline
\end{tabular}


Los mínimos y máximos de CL en relación a la edad oscilan entre 6 y 19 puntos, estando la escala comprendida entre 0 y 20 , por lo que se podría destacar que ningún alumno ha obtenido la máxima puntuación, algo que cabría haber esperado ya que el test de CL está optimizado para estudiantes de último ciclo de ESO. La media de competencia lectora en alumnos adolescentes de 15 años está en 13,7 puntos, mientras que la de los alumnos universitarios en 14,42 puntos. A mejor hábitos lectores mayor puntuación en competencia lectora $(2=17,65), 3,23$ puntos por encima de la media (tabla 3), independientemente de la edad.

Los alumnos de 18 años son los que mejor puntuación obtienen en competencia lectora con 14,88 puntos sobre 20. Los que peor puntuación obtienen son los alumnos de 24 años con 13,27 puntos sobre veinte en CL.

Tabla 4. Hábitos Lectores, Media de Inteligencia Emocional, Media de Competencia LectorA

\begin{tabular}{lll}
\hline HL & CL & IE \\
\hline Bajo & 12,89 & 4,44 \\
Medio & 14,13 & 4,89 \\
Alto & 17,65 & 5,62 \\
Total & 14,42 & 4,91 \\
& & \\
\hline
\end{tabular}

La IE fluctúa entre 5,62 y 4,44, siendo la media de 4.91 sobre 7. Los alumnos que presentan una mayor puntuación en inteligencia emocional (5,62 sobre 7) también marcan al alza significativamente en hábitos lectores logrando la máxima nota (2 de 2), así como obtienen los mejores resultados en competencia lectora (17,65 de 20). Esta proporción decrece paralelamente en los tres constructos, esto es, a menor inteligencia emocional peor competencia lectora en relación a los hábitos lectores; es decir, para los peores hábitos también puntuaciones más bajas tanto en competencia lectora como en inteligencia emocional.

\section{Discusión y conclusión}

La OCDE ya plantea que los alumnos con buen hábito lector puntúan sistemáticamente mejor en competencia lectora - cuestión que se corrobora en este estudio - al observarse cómo los alumnos universitarios que plantean ser lectores como forma de vida (que no leen por obligación, poseen libros que no son de texto o tienen un hábito lector afianzado) obtienen mejores calificaciones de manera general en competencia lectora. 
La hipótesis inicial del estudio queda demostrada ya que existe una relación entre los hábitos lectores con respecto a la inteligencia emocional y la competencia lectora. Así, para niveles de inteligencia emocional mayor también se obtienen mejores datos en competencia lectora ( $\mathrm{IE}=5,62$ con $\mathrm{CL}=17,65) \mathrm{y}$, al contrario, a menores niveles de competencia lectora peor puntuación en inteligencia emocional ( $\mathrm{IE}=4,44$ con $\mathrm{CL}=12,89$ ). Cabe destacar que son los alumnos más jóvenes (18 años) los que mejor puntuación en competencia lectora obtienen Esto puede ser debido a la competitividad que tienen que asumir los alumnos de Bachillerato de cara a selectividad que los obliga a leer y concentrarse para estudiar. Además, donde se relacionan los hábitos lectores con la inteligencia emocional, se confirma que también mantienen una estrecha relación puesto que los alumnos con una inteligencia emocional más alta son los que muestran un hábito lector más arraigado ( $\mathrm{HL}=2$ con $\mathrm{IE}=5,62)$ y viceversa $(\mathrm{HL}=0$ con $\mathrm{IE}=4,44)$. Es necesario resaltar que en IE, los apartados que han obtenido mejores puntuaciones giran en torno a la percepción propia de las emociones, no tanto a las ajenas. Así, los encuestados piensan o sienten que son felices, en general, que son buenos observadores y que tienen gran capacidad para animarse. Se muestra que existe una relación directa entre los universitarios andaluces que mejor entienden sus propios sentimientos y se sienten más felices y una competencia lectora con las puntuaciones más elevadas. Probablemente, esto sea debido a que la cultura influye en los ajustes emocionales de los individuos y en sus percepciones emocionales (Fernández-Berrocal et al., 2005), y a un buen funcionamiento interpersonal y psicológico de los individuos (Lizeretti y Rodríguez, 2011), aunque el hecho de que los lectores son más felices ya lo apunta un estudio de la Universidad de Roma (GeMS, 2015).

Por último, dados los resultados obtenidos, se puede afirmar que existe una relación directa entre los tres parámetros planteados, IE, CL y HL. Los datos apuntan a que no solo la competencia lectora está relacionada directamente con la IE, sino que los hábitos lectores juegan un papel fundamental en esta relación. Y, dado que la lectura observada es la lúdica, meramente literaria, los datos apuntan a que leer por placer literatura puede ser la forma natural de entrenar la competencia lectora, sin necesidad de realizar actividades específicas con textos literarios.

Cabe plantearse, para futuras investigaciones, qué aspectos concretos de la inteligencia emocional influyen en la competencia lectora o en la tendencia a crear de la lectura un hábito, así como si entrenando la competencia lectora se pueden obtener mejores puntuaciones en inteligencia emocional y viceversa, lo que podría implicar una mejora del rendimiento académico si los centros educativos reforzaran más el papel de los psicólogos como orientadores sobre rutinas colectivas de mejora de la IE. 
Asimismo, una ampliación de la muestra a nivel nacional posibilitaría una visión más amplia y generalizada de la relación entre hábitos lectores, inteligencia emocional y competencia lectora, con la intención de que dichos estudios trasciendan lo científico para crear herramientas de mejora escolar en la práctica.

\section{Referencias}

Calderón, A. y Quijano, J. (2010). Características de la comprensión lectora en estudiantes universitarios. Estudios Socio-Jurídicos, 12, 337-364.

Castellano, J. E. (2010). Inteligencia emocional y comprensión lectora en alumnos de sexto grado de primaria de la red $n^{\circ} 4$ distrito del Callao. (Tesis doctoral). Universidad San Ignacio de Loyola.

Cisneros, M., Olave G. y Rojas, I. (2012). Cómo mejorar la capacidad inferencial en estudiantes universitarios. Educación y Educadores 15, (1), 45-61.

Dávalos, M. T. y León, J. A. (2013). Inferencias emocionales durante la comprensión lectora. Un estudio sobre el efecto de la valencia mediante una tarea de decisión léxica. Uaricha. Revista de Psicología 10 (21), 111-121.

De Nóbrega, N. y Franco, G. (2014). Inteligencia emocional y actividad lectora en una escuela de 1er ciclo. INFAD Revista de Psicología, 5 (1), 159-166.

Domínguez, D. A. y Pérez, M. N. (2009). Internet y el hábito de la lectura en los universitarios. Innovación Educativa, 9 (49), 11-17.

Fernández-Berrocal P., Salovey, P., Vera, A., Extremera, N. y Ramos, N. (2005). Cultural influences on the relation between perceived emotional intelligence and depression. RIPS/IRSP, 18, 91-107.

Ferrando, M. (2006). Creatividad e inteligencia emocional: un estudio empírico de alumnos con altas habilidades. (Tesis doctoral). Universidad de Murcia.

García, B. (2011). Estudio de los hábitos lectores de los estudiantes de la Universidad Europea de Madrid. Ibersid 5, 99-107.

Gardner, H. (1993). Estructura de la mente: Teoría de las inteligencias múltiples. Nueva York: Harper Collins Publisher.

GeMS (2015). The hapiness of Reading. Roma: Universitá Roma Tre.

Gilardoni, C. (2006). Valoración del libro y mecanismos de acercamiento a la lectura en los estudiantes universitarios. Serie Bibliotecología y Gestión De La Información 16, 5-44.

Goleman, D. (2007). Inteligencia emocional. Barcelona: Kairós.

González, M. C., Roldán, C. Arráez, D., Gutiérrez, N., Segura, A. y Fernández, A. (2010). Eficiencia lectora: comparación alumnos universitarios de ciencias técnicas, ciencias experimentales y de letras y alumnos de EE. MM. (ciencias y letras). Jornadas sobre Innovación Docente y Adaptación EEES en las titulaciones Técnicas, 137-140.

Gutiérrez, M. E. y Fernández-Castillo, A. (2009). Inteligencia emocional e implicaciones en el contexto educativo. Alcance de los programas de intervención. 
Avances en el estudio de la Inteligencia Emocional. I Congreso Internacional de Inteligencia Emocional. Santander: Fundación Marcelino Botín.

Jiménez-Pérez, E. (2014). Comprensión lectora VS Competencia lectora: qué son y qué relación existe entre ellas. Revista Investigaciones Sobre Lectura, 1, 65-74.

Larrañaga, E. y Yubero, S. (2005). El hábito lector como actitud. El origen de la categoría de "falsos lectores". Ocnos, 1, 43-60.

Lizeretti, N. P. y Rodríguez, A. (2011). La inteligencia emocional en salud mental: Una revisión. Ansiedad y Estrés, 17, 233-253.

Llorens, A. C., Gil, L., Vidal-Abarca, E., Martínez, M., Mañá, A. y Gilabert, R. (2011). Prueba de competencia lectora para Educación Secundaria (ComLec). Psicothema, 23 (4), 808-817.

Moje, E. B., Overby, M., Tysvaer, N. y Morris, K. (2008). The complex world of adolescent literacy: myths, motivations and mysteries. Harvard Educational Review. 78 (1), 107-153.

Petit, M. (2003). El libro y la lectura: más allá del utilitarismo y el placer. El Bibliotecario, 3 (25), 13-26.

Ripoll, J. C. y Aguado, G. (2014). La mejora de la comprensión lectora en español: un meta-análisis. Revista de Psicopedagogía, 19 (1), 27-44.

Teberosky, A., Guàrdia, J. y Escoriza, J. (1996). Las prácticas de lectura en estudiantes universitarios. Anuario de Psicología, 70, 85-107.

VV. AA. (2011). Progress in International Reading Literacy Study 2011. IEA: Boston (EE. UU.).

VV. AA. (2011). Barómetro de lectura y compra de libros en Andalucía. Málaga, España: Observatorio de la Lectura en Andalucía.

VV. AA. (2012). Programme for International Student Assessment. París: OCDE.

Villardón-Gallego, L., Yániz, C., Achurra, C., Iraurgi, I. y Aguilar, M. (2013). Learning competence in university: development and structural validation of a scale of measure. Revista de Psicodidáctica, 18 (2), 357-374.

Wong, C. S. y Law, K. S. (2002). The effects of leader and follower emotional intelligence on performance and attitude: An exploratory study. The Leadership Quarterly, 13, 243274. 


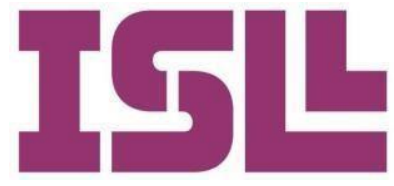

Númber 10

2018 December

\title{
Emotional intelligence as a predictor of reading habits and reading competence in university students
}

\author{
Elena Jiménez Pérez \\ Universidad de Jaén
}

Received: 01-07-2018

Accepted: 30-09-2018

Pag. 30 to 54

\section{Keywords}

Reading literacy, emotional intelligence, university students, Reading habits, Reading, natural method
In this study the relationship between emotional intelligence and reading skills from reading habit is analysed in a total of 1051 Andalusian university students to check if students score higher in EI are those with best results in reading literacy and if it's related to the consolidated reading habits. To carry out this research have been used tested tools: questionnaire MECD reading habits, reading proficiency test ComLec and test of Emotional Intelligence Wong and Lang. ICT has been the presentation of the test to students through a web link with exclusion criteria for those questionnaires that may be null. The results suggest that learners who score better, happier emotional intelligence as W y L, show a higher reading skill and understand reading as part of their lives, which can establish a natural relationship between the three parameters, and opens new horizons research to determine in more detail what that relationship and how to improve a training others. In conclusion, we can draw that the relationship between the reading habit, reading skills and emotional intelligence is directly proportional. 


\section{Introduction}

If you look at the reports of the Guild of Publishers of Spain, which indicates a decline in the purchase of books in general that does not break the negative trend of sales since 2008 , it should be noted that in the country every time you read fewer books. Thus, it is understood that more and more university professors are lamenting that each time they read less (García, 2011), a question that is admitted by the students themselves (Gilardoni, 2006) and, by extension, that reading comprehension it has not improved in the last 3 decades (Ripoll and Aguado, 2014) since there is a close relationship between reading habit and reading competence according to the OECD. University students' access to the already deficient degree to understand the texts (Calderón and Quijano, 2010) and other weaknesses in reading comprehension (Domínguez and Pérez, 2009), which go together with their low interest in reading in favor of other forms of entertainment (Gilardoni, 2006). This information contrasts with the official surveys of reading habits that have been carried out by the Andalusian Observatory of Reading (2011), for example, where $70.5 \%$ of the surveyed Andalusians aged between 14 and 24 years old say they read regularly. Possibly, more than half of the subjects distorts their image with the classic measures of reading by "the social desirability" of belonging to the reading class (Larrañaga and Yubero, 2005), since this can be understood as elitist because being a reader requires a certain level of self- demand and reading discipline.

But the university student, beyond his considerations, should be a good reader of truth (Cisneros, Olave and Rojas, 2012) and not use reading exclusively to read notes (Teberosky et al., 1996). And, above all, university students should be aware of if they understand what they really read (González, Roldán, Arráez, Gutiérrez, Segura and Fernández, 2010) so that they can discern if they should improve their reading competence.

It should be noted that adolescents with greater motivation towards reading, understood as a consolidated reading habit, have better reading skills and identify websites with useful information more quickly (Moje, Overby, Tysvaer and Morris, 2008). Also, people with better emotional management need less energy to process information (Dávalos and León, 2013), so there is the possibility of spending more time on other forms of leisure and, therefore, be happier.

The weight of emotional intelligence is very important in academic life (Gutiérrez and Fernández-Castillo, 2009), it has been demonstrated in studies (Petit, 2003, Castellano, 2010, De Nóbrega and Franco, 2014). Some authors suggest that emotional intelligence makes it possible to achieve an adequate performance in reading competence by how it relates to the regulation of perception through the understanding of feelings, the ability to learn according to mood (Villardón-Gallego, Yániz, Achurra, Iraurgi and Aguilar, 2013). What is really significant of the emotional intelligence for 
this study is that its correct management confers greater responsibility to the individual, better ability to concentrate, and to pay attention to the task that is being carried out, less impulsivity and better self-control (Goleman, 2007), all of them facilitators of the reading task. This, in part, could explain the relationship of the low rate of school failure in students with more established reading habits according to the OECD, if there is a relationship between the constructs of emotional intelligence and reading competence with reference to reading habits.

Regarding reading comprehension, understood as the ability of a human being to understand what an author wanted to say through a written text as objectively as possible (Jiménez, 2014), it is established that the process of understanding a text when reading It enlightens or stimulates emotions -either positive or negative- at almost the same time (Dávalos and León, 2013), so it is already theoretically pointed out that there is a relationship between understanding a text and the emotional intelligence of a subject. Without forgetting that the act of reading, as a natural way of training reading competence (Jiménez-Pérez, 2014), produces beneficial sensations in the organism (Dávalos and León, 2013) in the form of positive perception of emotions (Moje et al., 2008). Since there is a correlation between emotional and verbal intelligence (Ferrando, 2006), a direct relationship with reading competence would be explained, both closely related according to Gardner in his theory of multiple intelligences, that is, the ability of a being human being to use his reading comprehension in a useful way in the society that surrounds him (Jiménez-Pérez, 2014).

The main objective of this research is to perform a descriptive analysis of the distribution of emotional intelligence, on the one hand, and of reading competence, on the other, according to reading habits. Thus, as a hypothesis, we start from the possibility that students with more established reading habits should have better reading skills. Therefore, the possibility arises that the scores of the three parameters are linked in a directly proportional way. The hypothesis is the follow: check that there is a relationship between reading habits with respect to emotional intelligence and reading competence.

\section{Method}

\section{Participants}

In total, the 1051 students who have taken the test are divided according to sex in 474 men and 577 women, what gives a percentage of $45.09 \%$ men and $54.91 \%$ women. The fact that the number of women is higher may be due to the prevalence of teaching departments where teaching is taught in Bachelor's Degree, Primary and Secondary, where the number of women is higher than that of men. 
The test section of this study has been designed to facilitate access to questionnaires to the maximum number of students possible and to favor the work of the professors who have been willing to participate in this study, thus avoiding the use of traditional questionnaires in paper to expedite the procedures.

In addition, the respondents know if they have completed the questionnaire correctly and without incident because at the end a personal code is published confirming the conclusion of the global test and attests that the information provided has been successfully passed to the database.

To validate the questionnaires, there are several programmed nuances that automatically invalidate the test, causing the data that is recorded to not be passed on to the main database. To begin with, the logical time of each block is stipulated in advance by the base study that supports each test; For example, the emotional intelligence is estimated at 8 minutes, so any test that exceeds three times that time is canceled (an EI test of 24 minutes and up is automatically deleted, and with it, the rest of the binding information). In this case, the lower limit is indicated by a quarter, that is, any test that requires less than 2 minutes will not be taken into account. The test of reading habits requires a time of 15 minutes on average and that of reading competence 30 minutes, and both are subject to the same system of disqualification as the previous one. Finally, each test - divided into the three blocks indicated above (EI, RC and HR) - that yields a percentage of five repeated answers in a row exceeding 50\% is not stored in the database; similarly, it happens with blank answers. Each test computes the same percentage and prevents it from being saved in the final database.

\section{Instruments}

The final questionnaire has been raised based on three tests:

Tabla 1. Instruments for ítems evaluations

\begin{tabular}{ccc}
\hline RH $\left(15^{\prime}\right)$ & EI (8') & RC (30') \\
\hline Test of reading habits & Wong and Lang & Reading \\
of 2001 published by & Emotional Intelligence & Comprehension Test \\
the MECD & Test & ComLec \\
& & \\
\hline
\end{tabular}

The emotional intelligence test WONG and LANG

The full name of this test is Wong and Law Emotional Intelligence Scale (WLEIS) and is a basic self-report measure of Emotional Intelligence. The author is Dr. Chi-Sum Wong (Department of Management the Chinese University of Hong Kong 
Shatin, in Hong Kong, China). It lacks adaptations to Spanish and its description of the type of evaluation focuses on being a self-report measure composed of 16 items with a Likert scale of 7 points where, in general, the higher the score, the greater the emotional intelligence. Regarding the objectives of this evaluation, it is a record of emotional intelligence in the organizational field. The factors evaluated in this test are measured on a scale that is composed of four dimensions (four items per dimension): 1) Evaluation of one's own emotions; 2) Evaluation of the emotions of others; 3) Use of emotions, and 4) Regulation of emotions. The age to perform this test is from 16 years onwards while the administration time fluctuates between five and ten minutes. The psychometric data are operated through Cronbach's alpha and the internal consistency reported was that the evaluation of one's emotions amounted to $\alpha=0.87$, among which the perception of one's own happiness stands out; the evaluation of the emotions of others was positioned at $\alpha=$ 0.90 where the ability to empathize with others is established. Thus, the use of emotions in $\alpha=0.84$ as ability to positivize the day to day or manage stress; and the regulation of emotions in $\alpha=0.83$ as a form of self-control and self-knowledge.

\section{The ComLec reading competence test}

The ComLec test (Llorens, Gil, Vidal-Abarca, Martínez, Mañá and Gilabert, 2011) consists of five texts, three continuous and two discontinuous and a total of 20 questions prepared following the PISA 2000 parameters. The length of the texts varies from the 274 words to the 426 and are written mainly expository and argumentative, in relation to the continuous, and diagram and graph with or more than 130 discontinuous words.

The twenty questions are divided into information retrieval, integration, reflection on the content and form of the text. The response format is divided into multiple choices with four alternatives. The questions are of the open and closed type, the multiple choice prevailing because this test has been designed with the intention of programming it and thus facilitate its correction. For this reason, open questions would hinder this purpose because they cannot completely enclose certain types of questions from a computational linguistic point of view.

The psychometric data are also evaluated according to the Cronbach alpha coefficient where a global index of 795 was obtained.

The indices of difficulty of the items (values between, 25 and, 74) are shown and also the indices of homogeneity of the items appear (values between, 21 and, 48).

Despite the fact that this test has been designed for 15-year-olds, the texts and the questions that have been used are perfectly valid for higher age ranges since the type 
of texts and questions suggested by PISA have been used both for schoolchildren as for adults.

The approach of the test leaves the criterion of the subject whether to read the whole text to start or, on the contrary, the questions, for example. Therefore, students are asked to use the information in the text to answer the questions, leaving them the possibility to use their own strategies.

The proficiency test used raises a table of centiles thrown by the validity study of the project, which will be taken only as a reference for the present analysis. Therefore, even if the average obtained by 15 -year-old students is the starting point, this information will be defined by the average obtained by the university students themselves when they take the reading test.

On this selection an a2.3 has been created. The questionnaire of the MECD on Reactive Habits of the school population

The questionnaire of the Ministry of Education Science and Sports of Spain, generated in 2001, is composed of 50 questions, focused on a general public scattered throughout the Spanish territory (so it pays attention to other mother tongues) that covers more than 12,000 users surveyed, students enrolled as of 15 years and older.

The first 11 questions, through subsections that arithmetically expand the spectrum to analyze, collect personal information of students, from their age or their mother tongue to their internet use, number of bathrooms in their home or studies of parents to establish a profile -economic of the respondent.

From question number 12, the interest is focused on the academic circle of students (grades, subjects, expectations, school ...).

From question 15 to question 50, including its sub-items, the questionnaire focuses on analyzing the reading habit per se and in relation to other activities. It is the most important part of the survey in terms of obtaining objective information about the habit of reading and also probes the role that the teacher plays in inculcating this routine.

The entity in charge of carrying out the survey, the Ministry of Education, Science and Sport, which usually uses the INE (National Institute of Statistics), is one of the most solvent bodies in projecting, collecting and analyzing data that, on occasion, serves starting point for studies endorsed by the CSIC.

With respect to the categorization of reading habits, eight items have been selected from the same questionnaire (questions 13, 19, 21, 24, 28, 36, 50) that answer the following questions: how much do parents or guardians read? reading is a topic of daily discussion in the family environment, if the individual usually read in his spare 
time or dedicates it to other priority activities, how many books are in the house without counting the text (considered obligatory), if the main reason for The one that the respondent reads is the pleasure or another, and if he agrees with a series of affirmations corresponding to the reading. algorithm to obtain three types of reading habits: low, medium and high, reading for pleasure being the starting point (school, academic, etc. readings are ignored). These items have been evaluated positively when they show, at least, $50 \%$ of responses that tend to interest in reading in those issues that contemplate several subsections, for example, it is positively valued to choose "because I like" instead of "because I they force "in response to why they read.

In this way, the low reading habits correspond to those students who respond positively to 3 or fewer items of the 8 previously indicated, the average reading habits when they respond positively to 4 items and high when they respond positively to more than 5 items, of the selected ones.

\section{Procedures}

University students have been chosen starting from a logical base age, 18 years, the official age with which a non-repetitive student can access higher education and giving an amplitude of ten years for the current trend to not to finish the studies in the time of minimum graduation established by law, and by the tendency to complement the studies with languages, masters, etc.

Both sexes have been taken into account in the measurement of reading habits, emotional intelligence and reading competence but the tests of Erasmus students have been ruled out because of the probability of not being able to master the language enough.

This study is based on the research data collected in a questionnaire that has been published on the internet in 2014. The address has been open on the website http://testRC.comprensionlectora.es for more than 8 months with the intention of reaching to the university teaching staff that has collaborated for their students to carry out this questionnaire. The test has been composed of several blocks, from which have been extracted those corresponding to IE (emotional intelligence), HR (reading habits) and RC (reading competence), in addition to the block of personal data, which group a total of 203 questions. Of these 203 items:

-159 belong to the first questionnaire, which corresponds to the reading habits and which integrate the questions with their sub-sections. 
-16 to the second questionnaire, whose correspondence is emotional intelligence and is maintained without adaptations, so that in order to obtain the score, the original guidelines are respected.

-24 to reading competition, which also remains faithful to the original questionnaire.

-Items referring to age, sex, study center and level.

\section{Results}

In the first place, the mean values of age are analyzed with respect to reading habits. The average age of the university students is around 21 years old, with the youngest students reading best, with an average age of 20.97 .

Table 2. Reader Habit and Average Age

\begin{tabular}{llcccr}
\hline RH & Age & T. Desv. & Min & Max & $\%$ \\
Bajo & 21,65 & 1,729 & 18 & 24 & $31,7 \%$ \\
Medio & 21,08 & 1,625 & 18 & 24 & $57,0 \%$ \\
Alto & 20,97 & 1,579 & 18 & 24 & $11,3 \%$ \\
TOTAL & 21,24 & 1,673 & 18 & 24 & $100,0 \%$
\end{tabular}

If you look at the table, you can see that a majority of college students surveyed enter the category of average reading habit, with $57 \%$ of the total in score 1 . It is significant to note that only $11.5 \%$ have solid reading habits, since that it would be expected that the university students could present more consolidated reading habits. 
Tabla 3. Hábito Lector y Media de Competencia Lectora

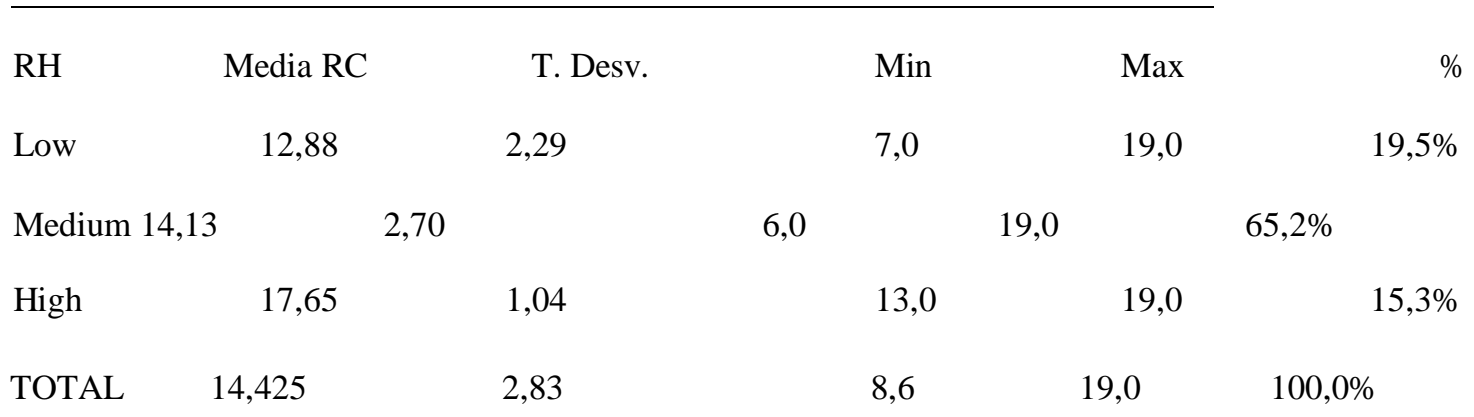

The minimums and maximums of RC in relation to age range between 6 and 19 points, with the scale between 0 and 20 , so it should be noted that no student has obtained the highest score, something that could have been expected since the RC test is optimized for students in the last cycle of ESO. The average of reading competence in adolescent students of 15 years is in 13,7 points, while that of the university students in 14,42 points. The better readers' habits, the higher score in reading competence $(2=17.65), 3.23$ points above the average (table 3 ), regardless of age.

The students of 18 years are those that better score in reading competition with 14.88 points out of 20 . Those who score worse are the students of 24 years with 13.27 points out of twenty in RC.

Table 4. Reader Habits, Emotional Intelligence Mean, Average Reading Competence

\begin{tabular}{lll}
\hline RH & RC & EI \\
\hline Low & 12,89 & 4,44 \\
Medio & 14,13 & 4,89 \\
Alto & 17,65 & 5,62 \\
Total & 14,42 & 4,91
\end{tabular}


EI fluctuates between 5.62 and 4.44, with an average of 4.91 out of 7. Students who have a higher score in emotional intelligence (5.62 out of 7) also mark up significantly in reading habits achieving the highest grade ( 2 of 2 ), as well as obtain the best results in reading competition (17.65 of 20). This proportion decreases in parallel in the three constructs, that is, the lower the emotional intelligence, the worse the reading competence in relation to reading habits; that is, for the worst habits also lower scores in both reading and emotional intelligence.

\section{Discussion and conclusion}

The OECD already states that students with good reading habits systematically score better in reading competence-a question that is corroborated in this study-when observing how university students who pose as readers as a way of life (who do not read by obligation, have books that they are not text or have a strong reader habit) they obtain better grades in a general way in reading competition.

The initial hypothesis of the study is demonstrated since there is a relationship between reading habits with respect to emotional intelligence and reading competence. Thus, for higher levels of emotional intelligence, better data is also obtained in reading competence $(\mathrm{EI}=5.62$ with $\mathrm{RC}=17.65)$ and, on the contrary, at lower levels of reading competence, worse score in emotional intelligence $(\mathrm{EI}=4,44$ with $\mathrm{RC}=12.89)$. It should be noted that it is the youngest students (18 years old) who have the best scores in reading skills.

This may be due to the competitiveness that students of Baccalaureate have to assume in the face of selectivity that forces them to read and concentrate to study. In addition, where the reading habits are related to emotional intelligence, it is confirmed that they also maintain a close relationship since students with a higher emotional intelligence are those who show a more ingrained reading habit $(\mathrm{HR}=2$ with $\mathrm{EI}=5.62)$ and vice versa $(\mathrm{HR}=0$ with $\mathrm{EI}=4.44)$. It is necessary to highlight that in $\mathrm{EI}$, the sections that have obtained better scores revolve around the perception of emotions, not so much those of others. Thus, respondents think or feel that they are happy, in general, that they are good observers and that they have a great capacity to be encouraged. It shows that there is a direct relationship between the Andalusian university students who understand their own feelings better and feel happier and a reading competence with the highest scores. Probably, this is due to the fact that culture influences the emotional adjustments of individuals and their emotional perceptions (Fernández-Berrocal et al., 2005), and the good interpersonal and psychological functioning of individuals (Lizeretti and Rodríguez, 2011), although the fact that readers are happier is already indicated by a study from the University of Rome (GeMS, 2015). 
Finally, given the results obtained, it can be affirmed that there is a direct relationship between the three proposed parameters, EI, RC and HR. The data suggest that not only reading competence is directly related to EI, but that reading habits play a fundamental role in this relationship. And, given that the reading observed is playful, merely literary, the data suggest that reading for pleasure can be the nat ural way to train reading skills, without the need to carry out specific activities with literary texts.

It should be considered, for future research, what specific aspects of emotional intelligence influence reading literacy or the tendency to create a habit of reading, as well as if training reading skills can obtain better scores in emotional intelligence and vice versa, that could imply an improvement of the academic performance if the educational centers reinforced more the role of the psychologists as guides on collective routines of improvement of the EI.

Likewise, an expansion of the sample at the national level would allow a broader and more generalized view of the relationship between reading habits, emotional intelligence and reading competence, with the intention that these studies transcend the scientific to create tools for school improvement in practice.

\section{References}

Calderón, A. y Quijano, J. (2010). Características de la comprensión lectora en estudiantes universitarios. Estudios Socio-Jurídicos, 12, 337-364.

Castellano, J. E. (2010). Inteligencia emocional y comprensión lectora en alumnos de sexto grado de primaria de la red $n^{\circ} 4$ distrito del Callao. (Tesis doctoral). Universidad San Ignacio de Loyola.

Cisneros, M., Olave G. y Rojas, I. (2012). Cómo mejorar la capacidad inferencial en estudiantes universitarios. Educación y Educadores 15, (1), 45-61.

Dávalos, M. T. y León, J. A. (2013). Inferencias emocionales durante la comprensión lectora. Un estudio sobre el efecto de la valencia mediante una tarea de decisión léxica. Uaricha. Revista de Psicología 10 (21), 111-121.

De Nóbrega, N. y Franco, G. (2014). Inteligencia emocional y actividad lectora en una escuela de 1er ciRCo. INFAD Revista de Psicología, 5 (1), 159-166.

Domínguez, D. A. y Pérez, M. N. (2009). Internet y el hábito de la lectura en los universitarios. Innovación Educativa, 9 (49), 11-17.

Fernández-Berrocal P., Salovey, P., Vera, A., Extremera, N. y Ramos, N. (2005). Cultural influences on the relation between perceived emotional intelligence and depression. RIPS/IRSP, 18, 91-107.

Ferrando, M. (2006). Creatividad e inteligencia emocional: un estudio empírico de alumnos con altas habilidades. (Tesis doctoral). Universidad de Murcia. 
García, B. (2011). Estudio de los hábitos lectores de los estudiantes de la Universidad Europea de Madrid. Ibersid 5, 99-107.

Gardner, H. (1993). Estructura de la mente: Teoría de las inteligencias múltiples. Nueva York: Harper Collins Publisher.

GeMS (2015). The hapiness of Reading. Roma: Universitá Roma Tre.

Gilardoni, C. (2006). Valoración del libro y mecanismos de acercamiento a la lectura en los estudiantes universitarios. Serie Bibliotecología y Gestión De La Información $16,5-44$.

Goleman, D. (2007). Inteligencia emocional. Barcelona: Kairós.

González, M. C., Roldán, C. Arráez, D., Gutiérrez, N., Segura, A. y Fernández, A. (2010). Eficiencia lectora: comparación alumnos universitarios de ciencias técnicas, ciencias experimentales y de letras y alumnos de EE. MM. (ciencias y letras). Jornadas sobre Innovación Docente y Adaptación EEES en las titulaciones Técnicas, 137-140.

Gutiérrez, M. E. y Fernández-Castillo, A. (2009). Inteligencia emocional e implicaciones en el contexto educativo. Alcance de los programas de intervención. Avances en el estudio de la Inteligencia Emocional. I Congreso Internacional de Inteligencia Emocional. Santander: Fundación Marcelino Botín.

Jiménez-Pérez, E. (2010). Una visión didáctica sobre la realidad de Miguel Hernández en Internet. Revista Islas, 165, 25-32.

Jiménez-Pérez, E. (2014). Comprensión lectora VS Competencia lectora: qué son y qué relación existe entre ellas. Revista Investigaciones Sobre Lectura, 1, 65-74.

Larrañaga, E. y Yubero, S. (2005). El hábito lector como actitud. El origen de la categoría de "falsos lectores". Ocnos, 1, 43-60.

Lizeretti, N. P. y Rodríguez, A. (2011). La inteligencia emocional en salud mental: Una revisión. Ansiedad y Estrés, 17, 233-253.

Llorens, A. C., Gil, L., Vidal-Abarca, E., Martínez, M., Mañá, A. y Gilabert, R. (2011). Prueba de competencia lectora para Educación Secundaria (ComLec). Psicothema, 23 (4), 808-817.

Moje, E. B., Overby, M., Tysvaer, N. y Morris, K. (2008). The complex world of adolescent literacy: myths, motivations and mysteries. Harvard Educational Review. 78 (1), 107-153.

Petit, M. (2003). El libro y la lectura: más allá del utilitarismo y el placer. El Bibliotecario, 3 (25), 13-26.

Ripoll, J. C. y Aguado, G. (2014). La mejora de la comprensión lectora en español: un meta-análisis. Revista de Psicopedagogía, 19 (1), 27-44.

Teberosky, A., Guàrdia, J. y Escoriza, J. (1996). Las prácticas de lectura en estudiantes universitarios. Anuario de Psicología, 70, 85-107. 
VV. AA. (2011). Progress in International Reading Literacy Study 2011. IEA: Boston (EE. UU.).

VV. AA. (2011). Barómetro de lectura y compra de libros en Andalucía. Málaga, España: Observatorio de la Lectura en Andalucía.

VV. AA. (2012). Programme for International Student Assessment. París: OCDE.

Villardón-Gallego, L., Yániz, C., Achurra, C., Iraurgi, I. y Aguilar, M. (2013). Learning competence in university: development and structural validation of a scale of measure. Revista de Psicodidáctica, 18 (2), 357-374.

Wong, C. S. y Law, K. S. (2002). The effects of leader and follower emotional intelligence on performance and attitude: An exploratory study. The Leadership Quarterly, 13, 243-274. 